\title{
Research of Standards to Interchangeable Manufacturing Processes' Classification and Coding
}

\author{
Wang Shaoni ${ }^{1, a}$ \\ ${ }^{1}$ Shandong Polytechnic, Jinan, 250104, Shandong, China \\ azni1978@126.com
}

Keywords: interchangeable manufacturing processes; classification; coding; standards

\begin{abstract}
Face classification method and line classification method are employed in manufacturing process classification, which is carried out based on investigation on interchangeable manufacturing processes. A classification draft of standards to manufacturing processes' classification is proposed, which lays the foundation for coding. This system is developed for effectively solving the problem of interchangeable manufacturing processes' knowledge communication and sharing in informationization process of mechanical manufacturing enterprises.
\end{abstract}

\section{Introduction}

Informationization is the trend of social development today. The informationization of manufacturing industry is complex system engineering, it is interdisciplinary, cross-industry and cross-domain. It cannot do without the support and guidance of standardization. The standard going first is a necessary condition and an important principle for the implementation of information technology [1].There is one of main symbols for powerful manufacturing country: Formatting technical standards and regulations system with the international advanced level, leads the formulation of international standard in some important fields [2].Standard is the main tool and weapon to cope with the global competition and cooperation, and protect our own rights and interests within WTO framework. But, there is no promulgated national standard for interchangeable manufacturing processes' classification and coding in our country. To fill this gap, we launched the subject "research of Standards to interchangeable manufacturing processes' classification and coding”.

\section{Significance of formulating the standards to interchangeable manufacturing processes' classification and coding}

Standard is unified provision for repeated things and conceptions. It is based on comprehensive results about science and technology and practical experience, negotiated by every aspects, approved by the competent bodies, and released from the specific form, thus as criterion and basis it shall be abided by. Standards to interchangeable manufacturing processes' classification and coding are unified provisions, which are the foundations of construction of process-knowledge management system. Perfecting and improving process-knowledge management system is the understructure of developing for manufacturing industry, furthermore the powerful manufacturing industry is footing stone of the construction and development of a nation. So, building standards to interchangeable manufacturing processes' classification and coding becomes the bottleneck of manufacturing industry, then it will influence national development.

Interchangeable manufacturing processes' classification and coding is basic work about constructing process-knowledge management system [3].

According to statistics, the amount of knowledge accumulated by human in recent 20 years is more than that in past 2000 years. Facing an ocean of knowledge, human feel feel it more and more difficult to manage and use knowledge. Faced with "knowledge explosion”, people built a variety of systems to manage and use knowledge. One of the basic work to construct knowledge management system is classification and coding. It lets the knowledges which have some common features stay together, distinguishing others. Then it sets a symbol code system, which can be identified and used by a computer. 
In the manufacturing enterprise, the classification and coding in the manufacturing process can make the processes more efficient and reasonable and it is more convenient to carry on further research, analysis and comparison. It will improve the efficiency and quality of the enterprise.

Interchangeable manufacturing processes' classification and coding is conducive to the realization of knowledge sharing and the operation between the systems [3].

Consistency about transmission and exchange among all kinds of process knowledge management systems is the premise and foundation to realization of the process of knowledge sharing. This consistency is based on the agreement in the various of process knowledge management systems.The classification and coding and standardization about process knowledge, can avoid misunderstanding and ambiguity, reduce one-name-multi-object, one-object-multi-name, and so on. It also can make things' (or concept's) name and term unified and standardized, guarantee description's uniqueness and reliability. Through the coding standards we can eliminate technical language barriers, and accelerate sharing and exchanging about interchangeable manufacturing processes' knowledge in the field of science.

Interchangeable manufacturing processes' classification and coding is an important prerequisite and guarantee of digital product.

For manufacturing industry, to define digital product entails describing all process knowledge which is involved in manufacturing effectively. With the development of computer technology, application of concurrent engineering and DFX, the information of the products is interchangeable in each link. Especially the emerging of network manufacturing and dynamic alliance makes the manufacturing unlimited in a region and manufacturing information and process knowledge should be shared on the network. This phenomenon requires inevitably that it is already extremely urgent that process knowledge should obtain the unification and standardization and classify and code the process knowledge.

\section{Domestic and international current situation of interchangeable manufacturing processes' classification and our draft}

Classification method in accordance with the material forming method, form of energy required, energy transfer medium and processing environment [4].

This method is recommended in late 1970s by professor J.Peclenic who worked for University of Birmingham. It classifies the process using 4 elements and their combination's similarity.

There are 5 material forming methods: the chip machining, deformation, adding material, chipless machining and material properties improving; 5 forms of energy required: mechanical energy, heat, electromagnetic energy, chemical energy and nuclear energy; 7 energy transfer mediums: solid, liquid, gas, mixed phase (such as solid and gas mixture), granular material, electromagnetic wave and sonic wave; 5 processing environments: in the gas, in the liquid, in the mixed phase, in vacuum and in the solid.

This method classifies the process using 4 elements and their combination's similarity, and it's more scientific. But it provides only the macroscopic classification, no the deeper level classification. It provides the possibility for technology innovation and the convenience for computer processing, and lays the foundation for the informatization and digitization of process.

Classification method in accordance with the energy and forming speed [4].

According to the level of the workpiece's surface forming speed, bounded by the speed of sound, processing methods fall into two categories: subsonic and supersonic. According to the level of the energy required, they fall into three categories: melting energy level (level I ), gasification energy level (level II ) and atomic binding forces destroyed energy level (level III)

Most of the traditional methods of cutting processing and plastic forming methods are in the level I , only the reaming and grinding are in the level II. But some new processing method developed in the 70's of last century, just as laser processing, electronic beam processing, ultrasonic machining and electrochemical machining, are in the level III basically. This method is also convenient to select the manufacturing methods and optimize the process. It also considers the energy factors of green 
manufacturing.

A method introduced by Japanese professor Hitomi katsundo [5].

This method is proposed in late 1970s, which divides interchangeable manufacturing processes into 7 categories: abnormal processing, deformation processing, connection processing, cutting, processing, assembly processing and auxiliary processing.

It involves widely, including abnormal processing, assembly processing and auxiliary processing.It's similar to the domestic classification method, easy to be accepted.But the classification tree formed from this method has not obvious systematicness. For example, it ties pressure processing and forging and extrusion in further division of deformation processing, and does not distinguish the subordinate relationship, not of the norm. Usually we think forging, extrusion belong to the category of pressure processing.

Classification method in accordance with the different ways of forming, it classifies processes from major attribute.

It divides interchangeable manufacturing processes into 8 categories: casting forming technology, plastic forming of metals, welding process, the forming of nonmetallic materials, machining, non-traditional machining, precision and ultra-precision machining technology and advanced manufacturing technology.

This classification method has its merits, but the division basis is not obvious, the principle is not clear.

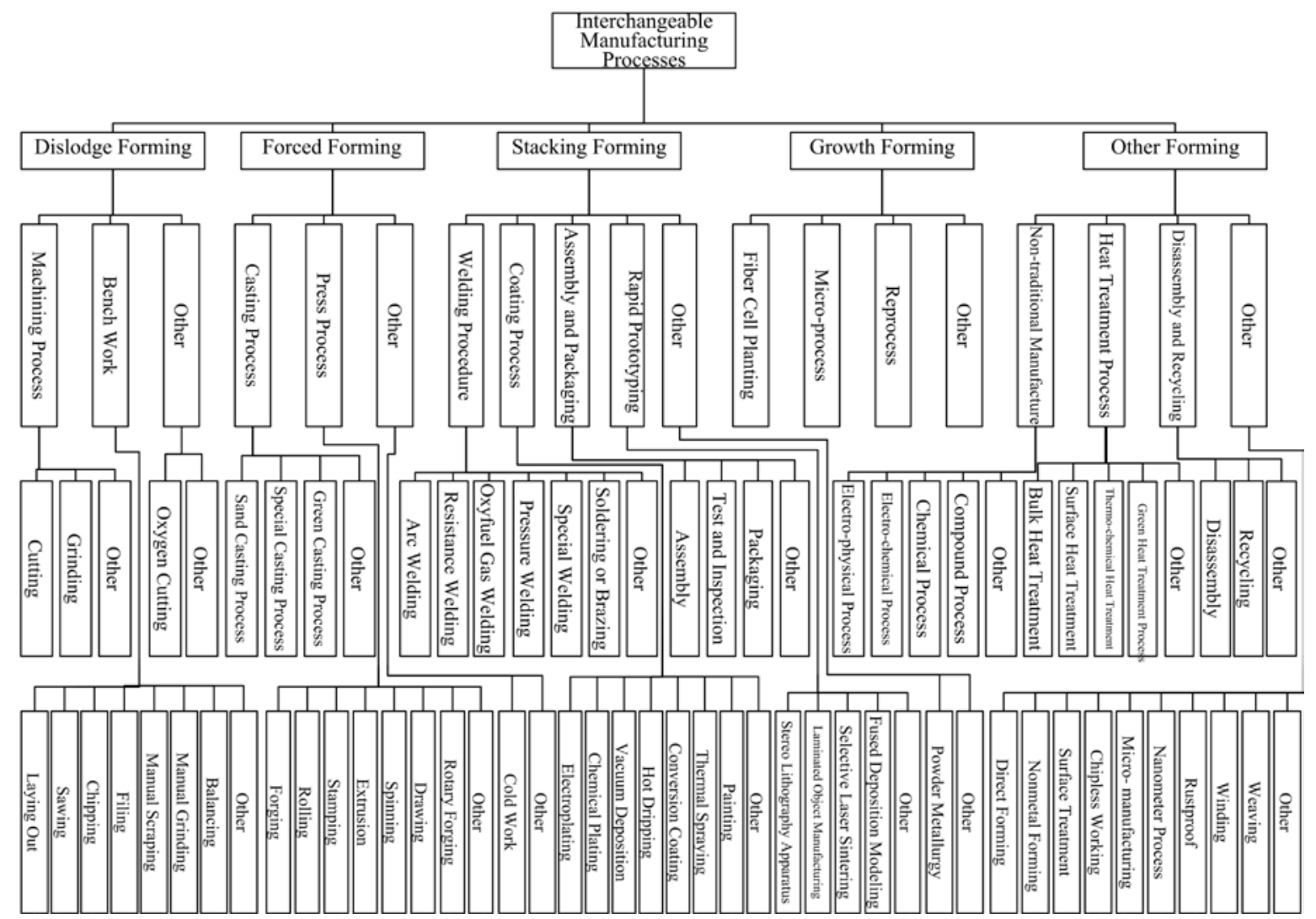

Fig. 1 Dendrogram of Classification draft about Interchangeable Manufacturing Processes (first three layers)

Our draft: "Classification of surface - line combination".

After analysis and comparison of the respective characteristics of the above 4 kinds of classification methods, combined with the view of modern forming, starting from the organization mode of material, we created "Classification of surface - line combination" draft. The characteristic is: use the surface classification, divide the first layer into 5 categories: dislodge forming, forced forming, stacking forming, growth forming and the other forming. Then , we adopt the line classification to get the further divisions on the above 5 categories layer by layer, until the completion of the fine classification. This method can promise: each layer will make room for future expansion.

Figure 1 is the first three layers of the draft; lack of space forbids the fourth and the fifth layer here.

Using the view of modern forming to divide the first layer, then refine the second, third, fourth 
layer based on the first. Every layer has clear principle of classification and there is a good scientificalness. It can be incorporated in the growth forming method and many new process methods (rapid prototyping, micro- manufacturing, nanometer process and so on), which adapts to the development of needs of the manufacturing sector. The draft contains "green cutting" and "removing and recovering”, emphasizes green environmental protection consciousness, and highlights the strategic thought of sustainable development.

\section{The coding draft of interchangeable manufacturing processes}

This draft is flexible classification and coding system, which consists of five parts (code segment), and can be expanded. The codes are represented by 7 Arabia numerals and the five code segments are as follows:

Code segment I: the first layer code, it consists of 1 digit, beginning from 0 , figure 0,1 to 9 available.

Code segment II: the second layer code, it consists of 1 digit, beginning from 0 , figure 0,1 to 9 available.

Code segment III: the third layer code, it consists of 2 digits, beginning from 00, figure 00, 01 to 99 available.

Code segment IV: the fourth layer code, it consists of 2 digits, beginning from 00 , figure 00,01 to 99 available.

Code segment V: the fifth layer code, it consists of 1 digit, beginning from 0 , figure 0,1 to 9 available.

In order to ensure a unified code, when the fourth layer do not subdivided downwards, " 0 " will be used to complete the following code until the seventh.The last code of each layer is the containing code, using the maximum code "9" or "99" expressed.Example and description (see figure 2)

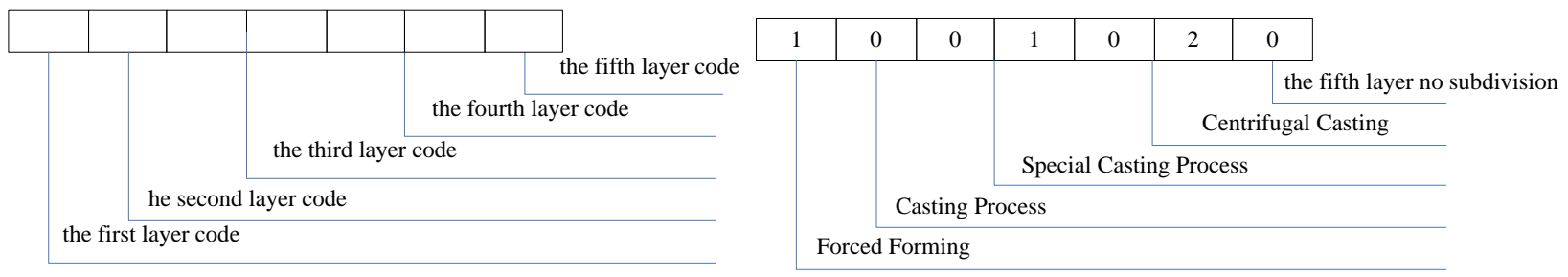

Fig. 2 Example and Description of the code about Interchangeable Manufacturing Processes

\section{Summary}

Classification and Coding about interchangeable manufacturing processes is an important prerequisite and guarantee for the standardization and unification of knowledge of processes. This paper presents the interchangeable manufacturing processes' classification and coding system, the work will lay the certain foundation for CIMS、PDM、ERP、AM and network manufacturing. The draft is based on extensive research on information at home and abroad, seeking the views of some domestic manufacturing enterprises. Many of the contents are just our ideas, and they need consultation with the relevant domestic departments. Welcome to put forward opinions and suggestions.

\section{References}

[1] Xue Hengming: The role of standards in national economic construction. Chinese Machine Tool Industry Association. Vol. 15-18 (2005), p4

[2] Wang Xiulun, Yu Xiaoyang and Huang Yongsheng: Advanced Manufacturing Technology (Dalian Jiaotong University, Dalian 2005).

[3] Wang Bingyi: Information Classification and coding (National Defence Industry Press, Beijing 2003).

[4] The manufacturing engineering department of Nanjing Aviation Institute of Aeronautical Compile: Machinery Manufacturing System (Shanghai Science and Technology Press, Shanghai 1984). 
[5] Hitomi Katsundo: Machinery Manufacturing System (Chinese Agricultural Machinery Press, Beijing 1983). 